\title{
Cancer Gene Therapy: vision and strategy for the new decade
}

\author{
Georgios Giamas Editor-in-Chief $\mathbb{D}^{1}$
}

Published online: 18 February 2020

(C) The Author(s), under exclusive licence to Springer Nature America, Inc. 2020

I would like to take this opportunity to thank and acknowledge Dr Steven K. Libutti's valuable leadership and contribution to the journal during the last 6 years. This is partly, but not solely, reflected in the consistent increase of manuscript submissions and publication of high quality research articles by various groups/laboratories worldwide. As a direct result, we have seen an increase in manuscripts submissions from 142 in 2017 to 202 in 2018 and 359 in 2019, our 2-year Impact Factor rose from 4.044 in 2018 to 4.681 in 2019, while the website receives over 250,000 views per year. (For more journal metrics, please refer to: https://www.nature.com/cgt/about).

I am aware that taking over the Editor-in-Chief (EIC) role of Cancer Gene Therapy (CGT) is a great honor as well as a huge responsibility especially when considering the highstandards and legacy that Steven left. I would like to reiterate our main goal to endorse and publish original, high-quality and cutting-edge articles that will promote the cancer research and treatment advances in the new decade. We also encourage submissions of inter- and multidisciplinary research manuscripts that fall under the general scope of CGT, including but not restricted to (i) basic science, (ii) bioengineering and biophysical approaches, (iii) bioinformatics analysis and mathematical modeling, in addition to (iv) translational studies, (v) systemic therapies and (vi) clinical trials.

I would also like to highlight and invite submissions for our 'Correspondence' article type, which provides a forum in the spirit of open scientific dialogue to comment and express opinions on scientific-related topics and on research studies published in previous issues of CGT or other journals.

Importantly, being aware of the inconvenience and frustration that long delays in the review process can cause to the authors, we are committed to significantly decrease the overall time for making an editorial decision. More specifically, we aim to decrease the average time to decision: (i) without external review to $\sim 7$ days (17 days currently) and (ii) following external review to $<30$ days (40 days currently). We also intend to make the whole editorial process more effective by including only a single revision round for most instances, ensuring of course that the quality threshold for publication is reached. As part of our strategy (a) to reduce the turnaround time for the editorial decisions and (b) to guarantee the appropriate assignment and evaluation of the submitted manuscripts to scientists with the respective expertise, we have also revamped our Editorial structure and appointed three new Associate Editors: Dr. Masatoshi Tagawa (Japan), Dr Steven Katz (USA) and Dr Michela Garofalo (UK).

Finally, we wish to further strengthen our social media presence and ask from all our authors and readers to follow us on our Twitter account (Cancer Gene Therapy; @ cgtnature) and help us promote the innovative research that is published in our journal.

As always, we welcome any suggestions and feedback of how we can improve, engage and sustain our international audience.
Georgios Giamas

g.giamas@sussex.ac.uk

1 Department of Biochemistry and Biomedicine, School of Life Sciences, University of Sussex, Brighton BN1 9QG, UK 